International Journal of Pure and Applied Mathematics

Volume 98 No. 2 2015, 261-273

ISSN: 1311-8080 (printed version); ISSN: 1314-3395 (on-line version)

url: http://www.ijpam.eu

doi: http://dx.doi.org/10.12732/ijpam.v98i2.8

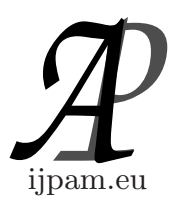

\title{
A NOTE ON A SPECIAL CLASS OF HERMITE POLYNOMIALS
}

\author{
C. Cesarano ${ }^{1 \S}$, C. Fornaro ${ }^{2}$, L. Vazquez ${ }^{3}$ \\ ${ }^{1,2}$ Università Telematica Internazionale Uninettuno \\ Rome, ITALY \\ ${ }^{3}$ Universidad Complutense de Madrid \\ Madrid, SPAIN
}

\begin{abstract}
This paper is devoted to the description of a special class of Hermite polynomials of five variables. It can be seen as an extension of the generalized vectorial Hermite polynomials of type $H_{m, n}(x, y)$ and at the same time as a generalization of the Gould-Hopper Hermite polynomials of type $H_{n}(x, y)$. We use the five-variable Hermite polynomials to derive reformulations of the well known operational relations satisfied from the generalized Hermite polynomials of different types.
\end{abstract}

AMS Subject Classification: $42 \mathrm{C} 05,33 \mathrm{C} 45,33 \mathrm{C} 47$

Key Words: Hermite polynomials, orthogonal polynomials, generating functions

\section{Introduction}

In a previous article (see [1]) we have discussed the generalized Hermite polynomials with two indices and two variables $H_{(m, n)}(x, y)$, which come from ordinary Hermite polynomials of type $H_{n}(x)$ (see $[2,3,4]$ ). We have then seen that the structure of polynomials $H_{(m, n)}(x, y)$ is based on the fact that a couple of indices act on a two-dimensional variable.

Received: October 11, 2014

(C) 2015 Academic Publications, Ltd.

$\S$ Correspondence author

url: www.acadpubl.eu 
We remind that, let the positive quadratic form:

$$
\begin{aligned}
q(x, y) & =a x^{2}+2 b x y+c y^{2} \\
a, c & >0 \\
a c-b^{2} & >0
\end{aligned}
$$

where $a, b, c$ are real numbers, the associated matrix reads:

$$
\hat{M}=\left(\begin{array}{ll}
a & b \\
b & c
\end{array}\right)
$$

and, since the (1), $|\hat{M}|>0$, that is an invertible matrix. Let now a vector $\underline{z}=\left(\begin{array}{l}x \\ y\end{array}\right)$ in space $\mathbb{R}^{2}$, it immediately follows that:

$$
\begin{aligned}
q(\underline{z}) & =\underline{z}^{t} \hat{M} \underline{z} \\
q(\underline{z}) & =\left(\begin{array}{ll}
x & y
\end{array}\right)\left(\begin{array}{ll}
a & b \\
b & c
\end{array}\right)\left(\begin{array}{l}
x \\
y
\end{array}\right)=a x^{2}+2 b x y+c y^{2}
\end{aligned}
$$

Definition 1. Let $\underline{z}=\left(\begin{array}{l}x \\ y\end{array}\right)$ and $\underline{w}=\left(\begin{array}{l}t \\ u\end{array}\right)$ be two vectors of space $\mathbb{R}^{2}$. We will name as two-index, two-variable Hermite polynomials $H_{m, n}(x, y)$ the polynomials defined by the following generating function:

$$
e^{\underline{z}^{t} \hat{M} \underline{w}-\frac{1}{2} \underline{w}^{t} \hat{M} \underline{w}}=\sum_{m=0}^{+\infty} \sum_{n=0}^{+\infty} \frac{t^{m}}{m !} \frac{u^{n}}{n !} H_{m, n}(x, y)
$$

By using the properties of matrix $\hat{M}$, in particular its invertibility, we can define the associated polynomials of $H_{m, n}(x, y)$. By noting in fact that the adjunct quadratic form of $q(\underline{z})$ writes:

$$
\bar{q}(\underline{z})=\underline{z}^{t} \hat{M}^{-1} \underline{z}
$$

we have that the two-index, two-variable associated Hermite polynomials $G_{m, n}(x$, $y$ ), are defined by the following generating function:

$$
e^{\underline{v}^{t} \hat{M}^{-1}} \underline{k}-\frac{1}{2} \underline{k} \hat{M}^{-1} \underline{k}=\sum_{m=0}^{+\infty} \sum_{n=0}^{+\infty} \frac{r^{m}}{m !} \frac{s^{n}}{n !} G_{m, n}(x, y)
$$

where $\underline{k}=\left(\begin{array}{l}r \\ s\end{array}\right)$ and $\left(\begin{array}{l}\xi \\ \eta\end{array}\right)$ such that $\underline{v}=\hat{M} \underline{z}$.

After manipulating the exponent of the 1.h.s, we can write the above relation in a more convenient form:

$$
e^{\underline{z}^{t} \underline{k}-\frac{1}{2} \underline{k} \hat{M}^{-1} \underline{k}}=\sum_{m=0}^{+\infty} \sum_{n=0}^{+\infty} \frac{r^{m}}{m !} \frac{s^{n}}{n !} G_{m, n}(x, y)
$$


The Hermite polynomials $H_{m, n}(x, y)$ and their associated $G_{m, n}(x, y)$ satisfy some important recurrence relations $[1,5]$, for instance:

$$
H_{m+1, n}(x, y)=(a x+b y) H_{m, n}(x, y)-a m H_{m-1, n}(x, y)-b n H_{m, n-1}(x, y)
$$

and:

$$
H_{m, n+1}(x, y)=(b x+c y) H_{m, n}(x, y)-b m H_{m-1, n}(x, y)-c n H_{m, n-1}(x, y)
$$

where $a, b, c$ are the real numbers defined in (1), and also:

$$
\frac{\partial}{\partial x} H_{m, n}(x, y)=a m H_{m-1, n}(x, y)+b n H_{m, n-1}(x, y)
$$

and

$$
\frac{\partial}{\partial y} H_{m, n}(x, y)=b m H_{m-1, n}(x, y)+c n H_{m, n-1}(x, y)
$$

Their proof can be viewed in a recent paper [6]. It is possible to simplify the operational techniques shown above by using a new class of Hermite polynomials.

\section{A Special Class of Hermite Polynomials}

In this section we present a special class if Hermite polynomials and we derive some relevant relations.

\section{Definition 2.}

We will call the polynomials defined by the following generating function as five-variable, two-index Hermite polynomials $H_{m, n}(x, y ; \xi, \eta \mid \chi)$ :

$$
e^{x t+y t^{2}+\xi \tau+\eta \tau^{2}+\chi t \tau}=\sum_{m=0}^{+\infty} \sum_{n=0}^{+\infty} \frac{t^{m}}{m !} \frac{\tau^{n}}{n !} H_{m, n}(x, y ; \xi, \eta \mid \chi)
$$

where $t$ and $\tau$ are continuous variables such that $|t|,|\tau|<+\infty$.

From the above definition, by exploiting the l.h.s., we can immediately obtain the explicit form of the polynomials $H_{m, n}(x, y ; \xi, \eta \mid \chi)$. In fact, by noting that $[3,7,8]$ :

$$
e^{x t+y t^{2}}, e^{\xi \tau+\eta \tau^{2}}
$$

are the generating functions of the two-variable Hermite polynomials of the type $H_{n}(x, y)$, we have:

$$
H_{m, n}(x, y ; \xi, \eta \mid \chi)=m ! n ! \sum_{q=0}^{\min (m, n)} \frac{\chi^{q} H_{m-q}(x, y) H_{n-q}(\xi, \eta)}{q !(m-q) !(n-q) !}
$$


Theorem 3. Hermite polynomials $H_{m, n}(x, y)$ can be written as $H_{m, n}(x, y$; $\xi, \eta \mid \chi)$, according to:

$$
H_{m, n}(x, y)=H_{m, n}\left(a x+b y,-\frac{1}{2} a ; b x+c y,-\frac{1}{2} c \mid-b\right)
$$

\section{Proof}

By manipulating the generating function of polynomials $H_{m, n}(x, y)$, given in (4), we can write:

$$
\begin{gathered}
\exp \left[\underline{z}^{t} \hat{M} \underline{w}-\frac{1}{2} \underline{w}^{t} \hat{M} \underline{w}\right]= \\
=\exp \left\{(a x+b y) t+(b x+c y) u-\frac{1}{2}[(a t+b u) t+(b t+c u) u]\right\}
\end{gathered}
$$

by rembering that $\underline{z}=\left(\begin{array}{l}x \\ y\end{array}\right)$ and $\underline{w}=\left(\begin{array}{l}t \\ u\end{array}\right)$ are two vectors of space $\mathbb{R}^{2}$ and:

$$
\hat{M}=\left(\begin{array}{ll}
a & b \\
b & c
\end{array}\right)
$$

with $a, b, c$ real numbers, such that:

$$
\begin{aligned}
a, c & >0 \\
a c-b^{2} & >0
\end{aligned}
$$

and then we obtain:

$$
\begin{gathered}
\exp \left[\underline{z}^{t} \hat{M} \underline{w}-\frac{1}{2} \underline{w}^{t} \hat{M} \underline{w}\right]= \\
\exp \left(a x t+b y t-\frac{1}{2} a t^{2}+b x u+c y u-\frac{1}{2} c u^{2}-b u t\right)
\end{gathered}
$$

We can rearrange the r.h.s. of the above equation in the following way:

$$
\begin{gathered}
\exp \left[\underline{z}^{t} \hat{M} \underline{w}-\frac{1}{2} \underline{w}^{t} \hat{M} \underline{w}\right]= \\
=\exp \left[(a x+b y) t-\frac{1}{2} a t^{2}\right] \exp \left[(b x+c y) u-\frac{1}{2} c u^{2}\right] \exp (-b u t)
\end{gathered}
$$

and by opportunity treating the variables in the exponential of the r.h.s., we write:

$$
\exp \left[\underline{z}^{t} \hat{M} \underline{w}-\frac{1}{2} \underline{w}^{t} \hat{M} \underline{w}\right]=
$$




$$
=\sum_{r=0}^{+\infty} \sum_{s=0}^{+\infty} \sum_{q=0}^{+\infty}(-1)^{q} \frac{t^{r+q}}{r !} \frac{u^{s+q}}{s !} \frac{b^{q}}{q !} H_{r}\left(a x+b y,-\frac{1}{2} a\right) H_{s}\left(b x+c y,-\frac{1}{2} c\right) .
$$

By setting:

$$
\begin{aligned}
& r+q=m \\
& s+q=n
\end{aligned}
$$

relation (19) can be written in a more convenient form:

$$
\begin{gathered}
\exp \left[\underline{z}^{t} \hat{M} \underline{w}-\frac{1}{2} \underline{w}^{t} \hat{M} \underline{w}\right]= \\
=\sum_{m=0}^{+\infty} \sum_{n=0}^{+\infty} t^{m} u^{n} \sum_{q=0}^{+\infty}\left[\frac{(-1)^{q} b^{q}}{q !(m-q) !(n-q) !} \cdot\right. \\
\left.\cdot H_{m-q}\left(a x+b y,-\frac{1}{2} a\right) H_{n-q}\left(b x+c y,-\frac{1}{2} c\right)\right]
\end{gathered}
$$

and from the (4), after equating the like $t$ and $u$ powers, we obtain the statement.

The opposite is not true, since the conditions:

$$
\begin{aligned}
& a c-b^{2}>0 \\
& a, c>0
\end{aligned}
$$

are not ensured.

It is also interesting to note that polynomials $H_{m, n}(x, y ; \xi, \eta \mid \chi)$ help us to write some interesting summing relations related to the generalized Hermite polynomials $[3,8]$. In fact, by setting:

$$
\begin{aligned}
& x=\xi \\
& y=\eta \\
& \chi=2 y
\end{aligned}
$$

the five-variable, two-index Hermite polynomials become:

$$
\begin{gathered}
H_{m, n}(x, y ; \xi, \eta \mid \chi)=H_{m, n}(x, y \mid 2 y)= \\
m ! n ! \sum_{q=0}^{\min (m, n)} \frac{(2 y)^{q} H_{m-q}(x, y) H_{n-q}(x, y)}{q !(m-q) !(n-q) !}
\end{gathered}
$$

and immediately follows from the identity

$$
H e_{n+m}(x, y)=m ! n ! \sum_{s=0}^{\min (n, m)}(2 y)^{s} \frac{H e_{n-s}(x, y) H e_{m-s}(x, y)}{(n-s) !(m-s) ! s !} .
$$


that

$$
H_{m, n}(x, y \mid 2 y)=H_{m+n}(x, y)
$$

In equation (6) we have introduced the two-index, two-variable associated Hermite polynomials $G_{m, n}(x, y)$ by their generating function [9] through the following relation:

$$
e^{\underline{z}^{t} \underline{k}-\frac{1}{2} \underline{k} \hat{M}^{-1} \underline{k}}=\sum_{m=0}^{+\infty} \sum_{n=0}^{+\infty} \frac{r^{m}}{m !} \frac{s^{n}}{n !} G_{m, n}(x, y)
$$

where $\underline{z}=\left(\begin{array}{l}x \\ y\end{array}\right)$ and $\underline{k}=\left(\begin{array}{l}r \\ s\end{array}\right)$ are the two vectors from the adjoint quadratic form

$$
\bar{q}(\underline{z})=\underline{z}^{t} \hat{M}^{-1} \underline{z}
$$

We can now explore how it is possible to represent the associated Hermite polynomials $G_{m, n}(x, y)$ in terms of the five-variable Hermite polynomials of the form $H_{m, n}(x, y ; \xi, \eta \mid \chi)$.

Corollary 4. Associated Hermite polynomials $G_{m, n}(x, y)$ can be written in ter of the five-variable, two-index Hermite polynomials $H_{m, n}(x, y ; \xi, \eta \mid \chi)$.

Proof. Expliciting the generating function of polynomials $G_{m, n}(x, y)$, we have:

$$
\begin{gathered}
\exp \left[\underline{z} \underline{k}-\frac{1}{2} \underline{k}^{t} \hat{M}^{-1} \underline{k}\right]= \\
\exp \left[\left(\begin{array}{ll}
x & y
\end{array}\right)\left(\begin{array}{c}
r \\
s
\end{array}\right)-\frac{1}{2}\left(\begin{array}{ll}
r & s
\end{array}\right)\left(\begin{array}{ll}
a & b \\
b & c
\end{array}\right)^{-1}\left(\begin{array}{c}
r \\
s
\end{array}\right)\right]
\end{gathered}
$$

where $\underline{z}=\left(\begin{array}{l}x \\ y\end{array}\right)$ and $\underline{k}=\left(\begin{array}{l}r \\ s\end{array}\right)$ with $x, y, r$, and $s$ are real numbers and matrix $\hat{M}=\left(\begin{array}{ll}a & b \\ b & c\end{array}\right)$ is invertible.

By manipulating the r.h.s of equation (21), we obtain:

$$
\begin{aligned}
& \exp \left[\left(\begin{array}{ll}
x & y
\end{array}\right)\left(\begin{array}{l}
r \\
s
\end{array}\right)-\frac{1}{2}\left(\begin{array}{ll}
r & s
\end{array}\right)\left(\begin{array}{cc}
a & b \\
b & c
\end{array}\right)^{-1}\left(\begin{array}{l}
r \\
s
\end{array}\right)\right]= \\
& =\exp \left[x r+y s-\frac{1}{2}\left(\begin{array}{ll}
r & s
\end{array}\right) \frac{1}{\Delta}\left(\begin{array}{cc}
c & -b \\
-b & a
\end{array}\right)\left(\begin{array}{c}
r \\
s
\end{array}\right)\right]= \\
& =\exp \left[x r+y s-\frac{1}{2 \Delta}\left(\begin{array}{cc}
c r-b s & -b r+a s
\end{array}\right)\left(\begin{array}{c}
r \\
s
\end{array}\right)\right]
\end{aligned}
$$


and then:

$$
\exp \left[\underline{z}^{t} \underline{k}-\frac{1}{2} \underline{k}^{t} \hat{M}^{-1} \underline{k}\right]=\exp \left[x r+y s-\frac{1}{2 \Delta} c r^{2}+\frac{1}{\Delta} b r s-\frac{1}{2 \Delta} a s^{2}\right]
$$

The expression on the r.h.s. of the above relation can be recast in a convenient form, by setting:

$$
\begin{gathered}
\exp \left[x r+y s-\frac{1}{2 \Delta} c r^{2}+\frac{1}{\Delta} b r s-\frac{1}{2 \Delta} a s^{2}\right]= \\
=\exp \left[x r+\left(-\frac{1}{2 \Delta} c\right) r^{2}\right] \exp \left[y s+\left(-\frac{1}{2 \Delta} a\right) s^{2}\right] \exp \left[\frac{1}{\Delta} b r s\right]
\end{gathered}
$$

The first two exponential on the r.h.s of the previous equation are the generating function of the generalized two-variable Hermite polynomials $H_{n}(x, y)$ $[2,3]$ and since the third exponential could be expanding in Taylor series, we get:

$$
\begin{aligned}
& \exp \left[\underline{z}^{t} \underline{k}-\frac{1}{2} \underline{k}^{t} \hat{M}^{-1} \underline{k}\right]= \\
& \sum_{m=0}^{+\infty} \frac{r^{m}}{m !} H_{m}\left(x,-\frac{1}{2 \Delta} c\right) \sum_{n=0}^{+\infty} \frac{s^{n}}{n !} H_{n}\left(y,-\frac{1}{2 \Delta} a\right) \sum_{q=0}^{+\infty} \frac{1}{q !} \frac{b^{q}}{\Delta} r^{q} s^{q}
\end{aligned}
$$

The r.h.s. of the above equation can be recast in the form:

$$
\sum_{m=0}^{+\infty} \sum_{n=0}^{+\infty} \sum_{q=0}^{+\infty} \frac{r^{m+q}}{m !} \frac{s^{n+q}}{n !} \frac{1}{q !} \frac{b^{q}}{\Delta} H_{m}\left(x,-\frac{1}{2 \Delta} c\right) H_{n}\left(y,-\frac{1}{2 \Delta} a\right)
$$

By setting $m+q=k$ and $n+q=j$, we can write:

$$
\sum_{k=0}^{+\infty} \sum_{j=0}^{+\infty} \sum_{q=0}^{+\infty} \frac{r^{k}}{(k-q) !} \frac{s^{j}}{(j-q) !} \frac{1}{q !} \frac{b^{q}}{\Delta} H_{k-q}\left(x,-\frac{1}{2 \Delta} c\right) H_{j-q}\left(y,-\frac{1}{2 \Delta} a\right)
$$

Without prejudicing the generality, we can set $k=m$ and $j=n$ and then:

$$
\begin{gathered}
\exp \left[\underline{z} \underline{\underline{k}}-\frac{1}{2} \underline{k}^{t} \hat{M}^{-1} \underline{k}\right]= \\
=\sum_{m=0}^{+\infty} \sum_{n=0}^{+\infty} \sum_{q=0}^{+\infty} \frac{r^{m}}{(m-q) !} \frac{s^{n}}{(n-q) !} \frac{1}{q !} \frac{b^{q}}{\Delta} H_{m-q}\left(x,-\frac{1}{2 \Delta} c\right) H_{n-q}\left(y,-\frac{1}{2 \Delta} a\right)
\end{gathered}
$$


In Definition 2 we have introduced the five-variable, two-index Hermite polynomials of the form $H_{m, n}(x, y ; \xi, \eta \mid \chi)$, that is:

$$
H_{m, n}(x, y ; \xi, \eta \mid \chi)=m ! n ! \sum_{q=0}^{\min (m, n)} \frac{\chi^{q} H_{m-q}(x, y) H_{n-q}(\xi, \eta)}{q !(m-q) !(n-q) !}
$$

We can observe that expression on r.h.s. of equation (28) can be recongnized as an Hermite polynomials $H_{m, n}(x, y ; \xi, \eta \mid \chi)$, by setting:

$$
\begin{aligned}
& x \rightarrow x \\
& y \rightarrow-\frac{c}{2 \Delta} \\
& \xi \rightarrow y \\
& \eta \rightarrow-\frac{a}{2 \Delta} \\
& \chi \rightarrow \frac{b}{\Delta}
\end{aligned}
$$

and then we can conclude that:

$$
\exp \left[\underline{z}^{t} \underline{k}-\frac{1}{2} \underline{k}^{t} \hat{M}^{-1} \underline{k}\right]=\sum_{m=0}^{+\infty} \sum_{n=0}^{+\infty} \frac{r^{m}}{m !} \frac{s^{n}}{n !} H_{m, n}\left(x,-\frac{c}{2 \Delta} ; y,-\frac{a}{2 \Delta} \mid \frac{b}{\Delta}\right)
$$

Since the generating function of the associate Hermite polynomials $G_{m, n}(x, y)$ has the form:

$$
e^{\underline{z}^{t} \underline{k}-\frac{1}{2} \underline{k} \hat{M}^{-1} \underline{k}}=\sum_{m=0}^{+\infty} \sum_{n=0}^{+\infty} \frac{r^{m}}{m !} \frac{s^{n}}{n !} G_{m, n}(x, y)
$$

we can easily obtain the thesis of the statement:

$$
G_{m, n}(x, y)=H_{m, n}\left(x,-\frac{c}{2 \Delta} ; y,-\frac{a}{2 \Delta} \mid \frac{b}{\Delta}\right)
$$

\section{Further Generalizations of Hermite Polynomials}

The operational results on the two-index, two-variable Hermite polynomials of type $H_{m, n}(x, y)$ and for their associate $G_{m, n}(x, y)$ in terms of the five-variable, two-index Hermite polynomials $H_{m, n}(x, y ; \xi, \eta \mid \chi)$, suggest us to generalize this special class of Hermite polynomials, to explore other relevant identities involving the Hermite polynomials of different type. Let us remember that the two-index, two-variable Hermite polynomials $H_{m, n}(x, y)$ have been introduced in Definition (1), through their generating function $[3,1]$, that is:

$$
e^{\underline{z}^{t} \hat{M} \underline{w}-\frac{1}{2} \underline{w}^{t} \hat{M} \underline{w}}=\sum_{m=0}^{+\infty} \sum_{n=0}^{+\infty} \frac{t^{m}}{m !} \frac{u^{n}}{n !} H_{m, n}(x, y)
$$


We can generalize the polynomials $H_{m, n}(x, y)$, by acting directly on the above expression.

\section{Definition 5.}

Let $\rho$ be a real number such that $|\rho|<0$ and let $\underline{z}=\left(\begin{array}{l}x \\ y\end{array}\right)$ and $\underline{w}=\left(\begin{array}{l}t \\ u\end{array}\right)$ be two vectors of space $\mathbb{R}^{2}$. We will call generalized two-index, two-variable Hermite polynomials $H_{m, n}(x, y ; \rho)$ the polynomials defined by the following generating function:

$$
e^{\underline{z}^{t} \hat{M} \underline{w}+\frac{1}{2} \rho \underline{w}^{t} \hat{M} \underline{w}}=\sum_{m=0}^{+\infty} \sum_{n=0}^{+\infty} \frac{t^{m}}{m !} \frac{u^{n}}{n !} H_{m, n}(x, y ; \rho)
$$

By exploiting the generating function on the above definition, we have:

$$
\begin{gathered}
\exp \left[\underline{z}^{t} \hat{M} \underline{w}+\frac{1}{2} \rho \underline{w}^{t} \hat{M} \underline{w}\right]= \\
=\exp \left[\left(\begin{array}{ll}
x & y
\end{array}\right)\left(\begin{array}{ll}
a & b \\
b & c
\end{array}\right)\left(\begin{array}{c}
t \\
u
\end{array}\right)+\frac{1}{2} \rho\left(\begin{array}{ll}
t & u
\end{array}\right)\left(\begin{array}{ll}
a & b \\
b & c
\end{array}\right)\left(\begin{array}{c}
t \\
u
\end{array}\right)\right]
\end{gathered}
$$

and by setting as $A$ the argument of the exponetial on the r.h.s. of the previous identity, we get:

$$
A=(a x+b y) t+(b x+c y) u+\frac{1}{2} \rho a t^{2}+\frac{1}{2} \rho u t b+\frac{1}{2} \rho t b u+\frac{1}{2} \rho c u^{2}
$$

It is immediate to note that the terms in the above relation could be recast in a convenient form, after remembering the structure of the generating function of the two-variable Hermite polynomials $H_{m}(x, y)$ [3]:

$$
\exp \left(x t+y t^{2}\right)=\sum_{n=0}^{+\infty} \frac{t^{n}}{n !} H_{n}(x, y)
$$

We have indeed:

$$
\begin{gathered}
\exp \left[\underline{z}^{t} \hat{H} \underline{w}+\frac{1}{2} \rho \underline{w}^{t} \hat{H} \underline{w}\right]= \\
=\exp \left[(a x+b y) t+\left(\frac{1}{2} \rho a\right) t^{2}\right] \exp \left[(b x+c y) u+\left(\frac{1}{2} \rho c\right) u^{2}\right] \exp (\rho b t u)
\end{gathered}
$$

and then, by expliciting the exponentials:

$$
\exp \left[\underline{z}^{t} \hat{H} \underline{w}+\frac{1}{2} \rho \underline{w}^{t} \hat{H} \underline{w}\right]=
$$




$$
=\sum_{m=0}^{+\infty} \frac{t^{m}}{m !} H_{m}\left(a x+b y, \frac{1}{2} \rho a\right) \sum_{n=0}^{+\infty} \frac{u^{n}}{n !} H_{n}\left(b x+c y, \frac{1}{2} \rho c\right) \sum_{q=0}^{+\infty} \frac{\rho^{q} b^{q}}{q !} t^{q} u^{q}
$$

that is:

$$
\begin{gathered}
\exp \left[\underline{z}^{t} \hat{H} \underline{w}+\frac{1}{2} \rho \underline{w}^{t} \hat{H} \underline{w}\right]= \\
=\sum_{m=0}^{+\infty} \sum_{n=0}^{+\infty} \sum_{q=0}^{+\infty} \frac{t^{m+q}}{m !} \frac{u^{n+q}}{n !} \frac{\rho^{q} b^{q}}{q !} H_{m}\left(a x+b y, \frac{1}{2} \rho a\right) H_{n}\left(b x+c y, \frac{1}{2} \rho c\right)
\end{gathered}
$$

By setting $m+q=k$ and $n+q=j$, we can rearrange the above expression in the form:

$$
\begin{gathered}
\exp \left[\underline{z}^{t} \hat{H} \underline{w}+\frac{1}{2} \rho \underline{w}^{t} \hat{H} \underline{w}\right]= \\
=\sum_{k=0}^{+\infty} \sum_{j=0}^{+\infty} \sum_{q=0}^{+\infty} \frac{t^{k}}{(k-q) !} \frac{u^{j}}{j !(j-q) !} \frac{\rho^{q} b^{q}}{q !} H_{k-q}\left(a x+b y, \frac{1}{2} \rho a\right) H_{j-q}\left(b x+c y, \frac{1}{2} \rho c\right)
\end{gathered}
$$

and by manipulating the terms involving the factorial, we end up with:

$$
\begin{gathered}
\exp \left[\underline{z}^{t} \hat{H} \underline{w}+\frac{1}{2} \rho \underline{w}^{t} \hat{H} \underline{w}\right]= \\
=\sum_{k=0}^{+\infty} \sum_{j=0}^{+\infty} \frac{t^{k}}{k !(k-q) !} \frac{u^{j}}{j !(j-q) !} . \\
\cdot \sum_{q=0}^{+\infty} \frac{k ! j !}{q !} H_{k-q}\left(a x+b y, \frac{1}{2} \rho a\right) H_{j-q}\left(b x+c y, \frac{1}{2} \rho c\right) \rho^{q} b^{q}
\end{gathered}
$$

that is:

$$
\begin{gathered}
\exp \left[\underline{z}^{t} \hat{H} \underline{w}+\frac{1}{2} \rho \underline{w}^{t} \hat{H} \underline{w}\right]= \\
=\sum_{k=0}^{+\infty} \sum_{j=0}^{+\infty} \frac{t^{k}}{k !} \frac{u^{j}}{j !} .
\end{gathered}
$$

$$
\cdot \sum_{q=0}^{\min (k, j)} q !\left(\begin{array}{c}
k \\
q
\end{array}\right)\left(\begin{array}{l}
j \\
q
\end{array}\right) H_{k-q}\left(a x+b y, \frac{1}{2} \rho a\right) H_{j-q}\left(b x+c y, \frac{1}{2} \rho c\right) \rho^{q} b^{q}
$$

Without prejudicing the generality, we can set $k=m$ and $j=n$.

$$
\exp \left[\underline{z}^{t} \hat{H} \underline{w}+\frac{1}{2} \rho \underline{w}^{t} \hat{H} \underline{w}\right]=
$$




$$
\begin{gathered}
=\sum_{m=0}^{+\infty} \sum_{n=0}^{+\infty} \frac{t^{m}}{m !} \frac{u^{n}}{n !} \sum_{q=0}^{\min (m, n)} \\
{\left[q !\left(\begin{array}{c}
m \\
q
\end{array}\right)\left(\begin{array}{c}
n \\
q
\end{array}\right) H_{m-q}\left(a x+b y, \frac{1}{2} \rho a\right) H_{n-q}\left(b x+c y, \frac{1}{2} \rho c\right) \rho^{q} b^{q}\right]}
\end{gathered}
$$

From Definition (3) we can conclude that the explicit form of the generalized two-index, two-variable Hermite polynomials of the type $H_{m, n}(x, y ; \rho)$ is given by the following relation:

$$
\begin{gathered}
H_{m, n}(x, y ; \rho)= \\
=\sum_{q=0}^{\min (m, n)} q !\left(\begin{array}{c}
m \\
q
\end{array}\right)\left(\begin{array}{c}
n \\
q
\end{array}\right) H_{m-q}\left(a x+b y, \frac{1}{2} \rho a\right) H_{n-q}\left(b x+c y, \frac{1}{2} \rho c\right) \rho^{q} b^{q}
\end{gathered}
$$

We note that the polynomials $H_{m, n}(x, y ; \rho)$ are also a generalization of the five-variable, two-index Hermite polynomials $H_{m, n}(x, y ; \xi, \eta \mid \chi)$ whose the explict form is stated in equation (13):

$$
H_{m, n}(x, y ; \xi, \eta \mid \chi)=m ! n ! \sum_{q=0}^{\min (m, n)} \frac{\chi^{q} H_{m-q}(x, y) H_{n-q}(\xi, \eta)}{q !(m-q) !(n-q) !}
$$

In fact, by setting $\eta=\rho$, we immediately obtain:

$$
H_{m, n}(x, y ; \rho)=H_{m, n}\left(a x+b y, \frac{1}{2} \rho a ; b x+c y, \frac{1}{2} \rho c \mid \rho b\right)
$$

The same considerations could be done relatively to the associate Hemrite polynomials of the form $G_{m, n}(x, y)$. We can in fact introduce the generalized two-index, two-variable Hermite polynomials by setting:

$$
e^{\underline{z}^{t} \underline{k}-\rho \underline{k} \hat{M}^{-1} \underline{k}}=\sum_{m=0}^{+\infty} \sum_{n=0}^{+\infty} \frac{r^{m}}{m !} \frac{s^{n}}{n !} G_{m, n}(x, y ; \rho)
$$

where, again, $\rho$ is a real number and $\underline{z}=\left(\begin{array}{l}x \\ y\end{array}\right)$ and $\underline{k}=\left(\begin{array}{l}r \\ s\end{array}\right)$ two vectors of the space $\mathbb{R}^{2}$.

By following the same procedure showed above, for the polynomials $H_{m, n}(x$, $y ; \rho)$, we can easily state the explicit form of the polynomials $G_{m, n}(x, y ; \rho)$ :

$$
G_{m, n}(x, y ; \rho)=
$$




$$
\sum_{q=0}^{\min (m, n)} q !\left(\begin{array}{c}
m \\
q
\end{array}\right)\left(\begin{array}{c}
n \\
q
\end{array}\right) H_{m-q}\left(x,-\frac{1}{\Delta} \rho c\right) H_{n-q}\left(y,-\frac{1}{\Delta} \rho a\right) \rho^{q} b^{q} .
$$

In Corollary 4 we have shown that the associate Hermite polynomials $G_{m, n}(x$, $y)$ can be written in terms of polynomials $H_{m, n}(x, y ; \xi, \eta \mid \chi)$ and we have just seen the same, relatively to the generalized Hermite ploynomials $H_{m, n}(x, y ; \rho)$; it is evident that we can represent the generalized associate Hermite polynomials of the form $G_{m, n}(x, y ; \rho)$ in terms of the five-variable, two-index Hermite polynomials $H_{m, n}(x, y ; \xi, \eta \mid \chi)$. In fact, by setting:

$$
\begin{aligned}
& x \rightarrow x \\
& y \rightarrow-\frac{c \rho}{\Delta} \\
& \xi \rightarrow y \\
& \eta \rightarrow-\frac{a \rho}{\Delta} \\
& \chi \rightarrow b \rho
\end{aligned}
$$

we can easily conclude with:

$$
G_{m, n}(x, y ; \rho)=H_{m, n}\left(x,-\frac{c \rho}{\Delta} ; y, \frac{a \rho}{\Delta} \mid \rho b\right)
$$

The results shown above point out the relevant contribution of this new class of Hermite polynomials. It is important to note that the vectorial Hermite polynomials are very useful in description of the quantum treatment of coupled harmonic oscillator; the five-variable Hermite polynomials presented in this paper could simplify the operational relation satisfied by vectorial Hermite polynomials and many other classes of orthogonal polynomials.

Then, since it is possible to represent the Generalized two-variable Bessel functions in terms of two-variable Hermite polynomials [10, 11, 12, 13], in a forthcoming paper we will investigate how it is possible to obtain different expressions of the above results by using special families of Bessel functions.

\section{References}

[1] C. Cesarano, A note on generalized Hermite polynomials, Int. J. of applied Math. and Informatics, 8 (2014), 1-6.

[2] P. Appell and J. Kampé de Fériet, Fonctions hypergéométriques et hypersphériques. Polynômes d'Hermite, Gauthier-Villars, Paris (1926).

[3] H.W. Gould, A.T. Hopper, Operational formulas connected with two generalizations of Hermite Polynomials, Duke Math. J., 29 (1962), 51-62. 
[4] G. Dattoli and C. Cesarano, On a new family of Hermite polynomials associated to parabolic cylinder functions, Applied Mathematics and Computation, 141 (2003), 143-149.

[5] G. Dattoli, A. Torre, S. Lorenzutta and C. Cesarano, Generalized polynomials and operatorial identities, Accad. Sc. di Torino Atti Sc. Fis., 132 (2000), 231-249.

[6] C. Cesarano, C. Fornaro and L. Vazquez, Operational results in biorthogonal Hermite functions, Acta Mathematica Uni. Comenianae, submitted for publication (2014).

[7] G. Dattoli, S. Lorenzutta, C. Cesarano, Bernstein polynomials and operational methods, Journal on Computation Analysis and Applications, 8 No. 4 (2006), 369-377.

[8] C. Cesarano, G.M. Cennamo and L. Placidi, Humbert Polynomials and Functions in Terms of Hermite Polynomials Towards Applications to Wave Propagation, WSEAS Transactions on Mathematics, 13 (2014), 595-602.

[9] H.M. Srivastava, H.L. Manocha, A treatise on generating functions, Wiley, New York (1984).

[10] C. Cesarano, D. Assante, A note on Generalized Bessel Functions, International Journal of Mathematical Models and Methods in Applied Sciences, $7(6)$ (2013), $625-629$.

[11] D. Assante, L. Verolino, Efficient evaluation of the longitudinal coupling impedance of a plane strip, Progress In Electromagnetics Research, 26 (2012), $251-265$.

[12] D. Assante, D. Davino, S. Falco, F. Schettino, L. Verolino, Coupling impedance of a charge traveling in a drift tube, IEEE transactions on magnetics, 41(5) (2005), $1924-1927$.

[13] D. Assante, C. Cesarano, Simple semi-analytical expressions of the lightning base current in the frequency-domain, Journal of Engineering Science and Technology Review, 7 (2014), 1 - 6. 
